\title{
Endonasal Approaches to Maxillary Sinus
}

\author{
${ }^{1}$ Carl Philpott, ${ }^{2}$ Anshul Sama
}

\begin{abstract}
Operating on the maxillary sinus has been a part of the otorhinolaryngologist's surgical repertoire being perceived as the most accessible sinus. Following the advent of endoscopic sinus surgery and advances in angled endoscopes and surgical instruments, approaches to the maxillary sinus have evolved leaving open approaches largely redundant. This review article will guide the readers through the key anatomical principles and techniques available for endoscopic approaches to the maxillary sinus.
\end{abstract}

Keywords: Antrostomy, Endonasal, Endoscopic, Maxiilary sinus. How to cite this article: Philpott C, Sama A. Endonasal Approaches to Maxillary Sinus. Int J Head Neck Surg 2018;9(1):26-31.

Source of support: Nil

Conflict of interest: None

\section{BACKGROUND}

Since the conception of endoscopic sinus surgery as delineated by Walter Messerklinger and his disciples using the Hopkins rod, the last three decades have seen the place of endoscopic sinus surgery become the standard of care. Resultantly, open approaches to the maxillary sinus are now largely rare. Operations like the Caldwell-Luc and lateral rhinotomy are relatively invasive and associated with not insignificant morbidity and do not respect the natural physiology of the sinuses. Even when considering surgery for sinonasal tumors where the functionality of the sinuses is not the prime objective, many options exist for endoscopic approaches that can avoid the traditional open approaches.

Functional endoscopic sinus surgery (FESS) was first introduced by Prof Heinz Stammberger who popularized the Messerklinger technique ${ }^{1}$ and it is the maxillary sinus that is most commonly addressed in the setting of inflammatory sinonasal disease, such as chronic rhinosinusitis (CRS). In the UK Sinonasal Audit of 2001, $18 \%$ of procedures involved the maxillary sinus, as compared with $7 \%$ involving the frontal sinus. ${ }^{2}$ However, the findings in revision surgery may suggest that it is also an area that

\footnotetext{
${ }^{1,2}$ Professor

${ }^{1}$ Norwich Medical School, University of East Anglia, Norwich England, UK

${ }^{2}$ Nottingham University Hospitals NHS Trust, Nottingham England, UK

Corresponding Author: Carl Philpott, Professor, Norwich Medical School, University of East Anglia, Norwich England, UK, e-mail: C.Philpott@uea.ac.uk
}

is perhaps inadequately addressed, especially when $0^{\circ}$ endoscopes only are used for surgery. ${ }^{3}$

Most recently, we have seen the advent of balloon sinuplasty and it has become a commonly available tool for clinic-based procedures, initially to the maxillary sinus, ${ }^{4}$ but with a more recent shift toward the frontal sinus. $^{5}$ This chapter will explore the range of endoscopic techniques currently in use by otorhinolaryngologists in addressing the maxillary sinus.

\section{TECHNIQUE}

\section{Balloon Sinuplasty}

Balloon sinuplasty has become an established minimally invasive endoscopic treatment for inflammatory sinus disease. It aims to widen the sinus ostia with minimal mucosal damage and can be used in isolation or in combination with more traditional endoscopic techniques. ${ }^{6}$ In its infancy, the technique required fluoroscopy and image intensifiers in an operating theatre setting. However, once the lighted guidewire devices became available, the technique became more accessible and is now frequently used in an ambulatory setting. Inflation of the balloon gently displaces, microfractures, and molds the bone surrounding the sinus outflow; the pressure achieved in the balloon is approximately $12 \mathrm{~atm}$. Dilatation of the maxillary ostium inherently involves dilatation of the ethmoid infundibulum and medial displacement of the free edge of the uncinated process, which remains intact. This allows drainage and port for irrigation of the maxillary sinus. However, if the goal of the intervention is to improve the delivery of topical medications to the maxillary sinus, this technique with intact uncinate process fails to deliver. A recent systematic review by the Cochrane group on the efficacy of this technique concluded that it did not demonstrate any superiority over conventional techniques. However, since this review in 2011, the REMODEL trial provided supportive evidence for maxillary sinus balloon sinuplasty ${ }^{8}$ with evidence accumulating in other studies $^{9,10}$ and an increase in the use of the technique seen especially in the USA as a clinic-based procedure ${ }^{5}$; it is likely that the technique will continue to be part of established practice for the foreseeable future.

\section{Middle Meatal Antrostomy and Maxillary Sinusotomy}

\section{Uncinectomy and Infundibulotomy}

Common terminology in use for the standard approach to the maxillary sinus is the middle meatal antrostomy. 
However, in cases of CRS, especially in those without nasal polyps, a widening of the maxillary sinus ostium may not be needed. If the goal of surgery is to establish drainage, irrigation of maxillary contents, or improve delivery of therapeutic agents to the sinuses, such as intranasal corticosteroids, exposure of the natural ostium may be all that is necessary. The key steps that are therefore required are uncinectomy and the resultant infundibulotomy, as the ethmoid infundibulum lies lateral to the free edge of the uncinate process. As the natural ostium of the maxillary sinus lies anterior and lateral to the free edge of the uncinate process, it is not normally visible with a $0^{\circ}$ endoscope. Dissection and removal of the entire uncinate process allow visualization of the natural maxillary ostium, especially with an angled endoscope. In addition, when both the mucosa and bone of the uncinate are diseased, residual tissue may result in persistent symptoms and prevent adequate medication delivery to the ostiomeatal complex. The variations in uncinate anatomy should be considered by using radiological examination before resection, with particular reference to its superior attachment and its relationship to the orbit. ${ }^{11}$ Figure 1 demonstrates the three common sites of attachment of the uncinate process:

- Type I: Lamina papyracea leading to the formation of a recessus terminalis

- Type II: Skull base/ethmoid "roof"

- Type III: Middle turbinate

In a recent study of anatomical findings at revision surgery for CRS, $64 \%$ of patients had a residual uncinate process; this was in keeping with a radiological study looking at findings from previous revision endoscopic sinus surgery (ESS). ${ }^{12}$ Figure 2 shows an example of a residual uncinate process in the presence of continued inflammatory changes (red arrow).

There are a number of varying techniques for the removal of the uncinate process that largely fall into

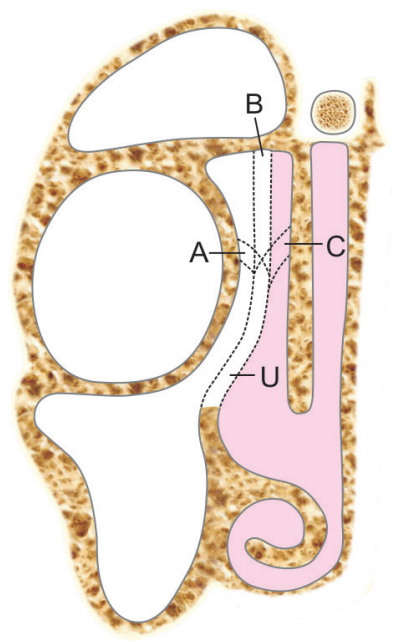

Fig. 1: The possible superior insertions of the uncinate process $(U)$; $\mathrm{A}=$ lamina papryacea, $\mathrm{B}=$ skull base, $\mathrm{C}=$ middle turbinate anterograde and retrograde dissection. Those advocates for the retrograde technique will consider that this avoids a blind entry through the lamina papyracea and thus the potential for consequent orbital injury. The retrograde or "back-to-front" approach is usually undertaken by using a backbiter (pediatric if required) to divide the uncinate at the junction of the inferior third and superior two thirds. The superior component can then be dissected and removed, for example, with a pediatric $90^{\circ}$ forceps or through cutting forceps. The inferior component, at its junction with the inferior turbinate, can then be separately removed with a downbiting antral punch or filleted out in its submucosal plane. For the anterograde approach, proponents typically use a sickle knife or Freer's elevator to divide the uncinate process from the lateral wall at is site of fibrous attachment with the lacrimal bone. Whichever method is chosen, removal should be sufficient to undertake the next step; if exposure of the maxillary sinus ostium is the only goal in a specific case, removal of only the lower $1 / 3$ of the uncinate may be adequate, as this still results in an adequate infundibulotomy for this goal.

As the uncinate process is often considered the doorway to the sinuses, failure to perform this step correctly may well result in poorer ESS outcomes. The uncinate process was described by Messerklinger as the first of four constant lamellae or landmarks when conducting ESS (Fig. 3, label 1).

Due to the anterolateral position of the natural sinus maxillary ostium relative to the uncinate process, it will not be possible to see the ostium without its removal in most cases. Correctly locating the natural maxillary ostium is paramount in avoiding false surgical ostium formation and therefore goes hand in hand with the uncinectomy. In the aforementioned recent series, $47 \%$ of the patients were found to have an incorrectly placed antrostomy and with $64 \%$ having a residual uncinate process, this is therefore not surprising.

Creation of a false surgical ostium risks mucociliary recirculation as a result of mucus flowing out of the natural ostium and in through the surgical neo-ostium ${ }^{13}$ (Fig. 4).

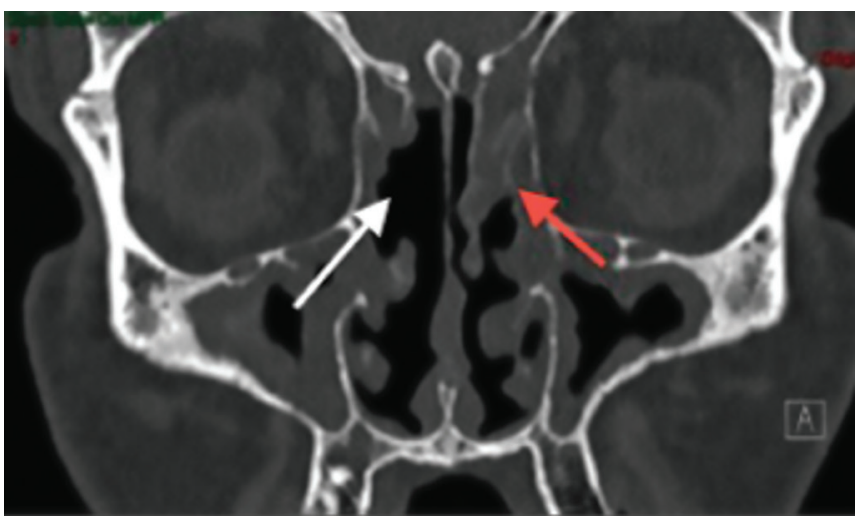

Fig. 2: Residual uncinate process (red arrow) and denuded middle turbinate (white arrow) in a patient presenting with recurrent disease following previous sinus surgery 


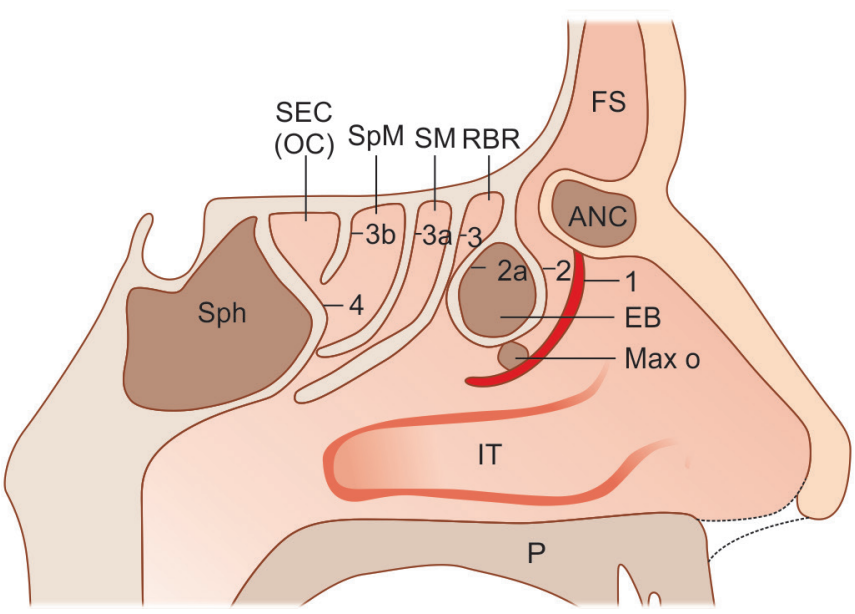

Fig. 3: The "constant" lamellae and inconsistent lamellae as described by Messerklinger. Sagittal section diagram; 1 = uncinate process, 2 = anterior wall of ethmoid bulla, 3 = basal lamella of middle turbinate, $4=$ face of sphenoid, ANC = agger nasi cell, $\mathrm{EB}=$ ethmoid bulla, max $\mathrm{o}=$ maxillary sinus ostium, $\mathrm{IT}=$ inferior turbinate, $2 \mathrm{a}=$ posterior wall of ethmoid bulla, $\mathrm{RBR}=$ retrobullar recess, $\mathrm{SM}=$ superior meatus, $3 \mathrm{a}=$ superior turbinate, $3 \mathrm{~b}=$ supreme turbinate (when present), SEC = sphenoethmoidal cleft (olfactory cleft), $\mathrm{Sph}=$ sphenoid, $\mathrm{P}=$ palate

Once the uncinate is properly removed, the best strategy is to use an angled endoscope to enable clear visualization of the natural ostium; using the angled scope can also be very helpful during the lower uncinectomy. A $30^{\circ}$ scope can be used to remove the lower uncinate and visualize the natural ostium; however, as can be seen in Figure 5, on occasions, a $70^{\circ}$ scope may even be necessary to avoid mistaking an accessory ostium for the natural one.

\section{Maxillary Sinusotomy}

In the presence of an accessory ostium, need for instrumentation within maxillary sinus (e.g., removal of antrochoanal polyp), and certain specific pathological causes of CRS (e.g., cystic fibrosis, Samter's triad, mycetoma, etc.), there is a need for extending the natural ostium of the maxillary sinus. In these situations, the natural ostium is extended posteriorly and inferiorly to a varying degree. Type I maxillary sinusotomy is when natural ostium is extended to posterior fontanelle to join the accessory ostium and usually extends the natural ostium by $1 \mathrm{~cm}$ or so. Type III maxillary sinusotomy is when the ostium is extended to the back wall of the maxillary sinus posteriorly, inferiorly to the base of the inferior turbinate, and anteriorly to the lacrimal sac. The need for the latter is limited and discussed further below. A type II maxillary sinusotomy lies in between these two. ${ }^{14}$

\section{Large Antrostomy}

There is ongoing debate regarding posterior enlargement of the maxillary antrostomy. Detractors of this approach will cite the main concern as being the drying effect of nasal airflow on the sinus mucosa that may in turn

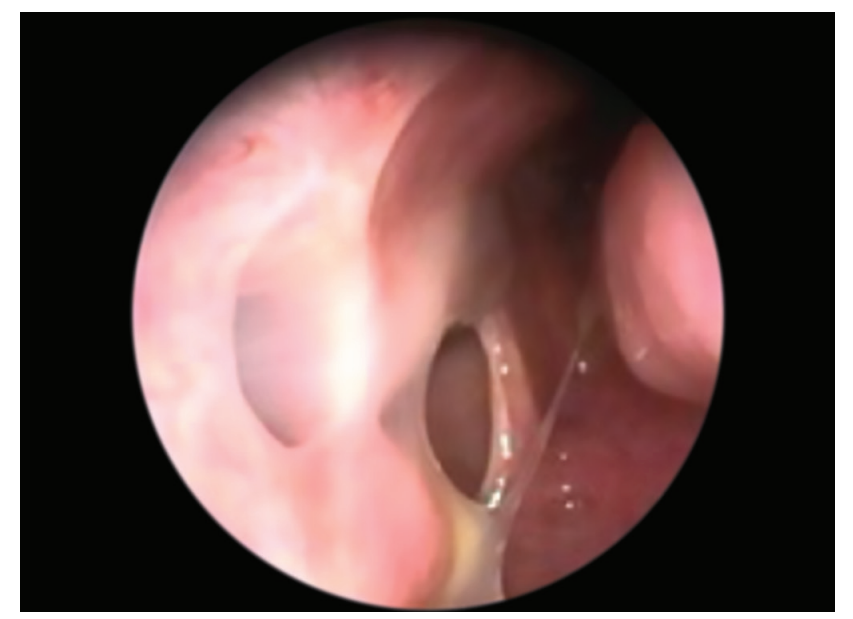

Fig. 4: Recirculation of mucus between a natural maxillary ostium and a posterior surgical antrostomy

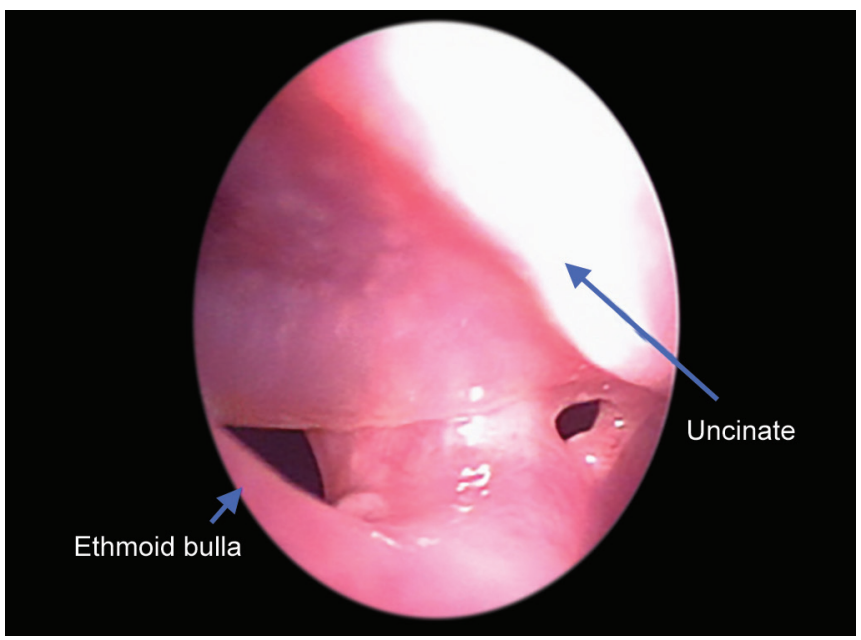

Fig. 5: View of the right ethmoid infundibulum with a $70^{\circ}$ endoscope showing the natural ostium anteriorly and an accessory ostium posteriorly

predispose to biofilm formation. ${ }^{13}$ The other major concern being the loss of protective nitric oxide concentration within the maxillary sinus. ${ }^{15}$ The posterior aspect of the ostium and mucosa in the ethmoid infundibulum posteriorly should ideally be preserved to avoid impairment of mucociliary clearance. In the aforementioned case series, an oversized antrostomy was demonstrated in $29 \%$ of patients, where it accounted for $>50 \%$ of the medial maxillary wall (Fig. 6). In some cases of CRS, especially in those with extensive nasal polyposis and allergic fungal rhinosinusitis, widening the natural ostium will aid clearance of the sinus contents at ESS. This can, however, be achieved with preservation of the posterior aspect on the ostium and infundibulum by inferior enlargement. Other examples are antrochoanal polyps, where removal of the stalk and root is key in avoiding recurrence and fungal balls where the pathology has often already distorted the normal anatomy, but a larger opening will be needed to clear the troublesome contents.

It is clear that there are polar opinions on this matter. Hwang and his team reported their successful series of 


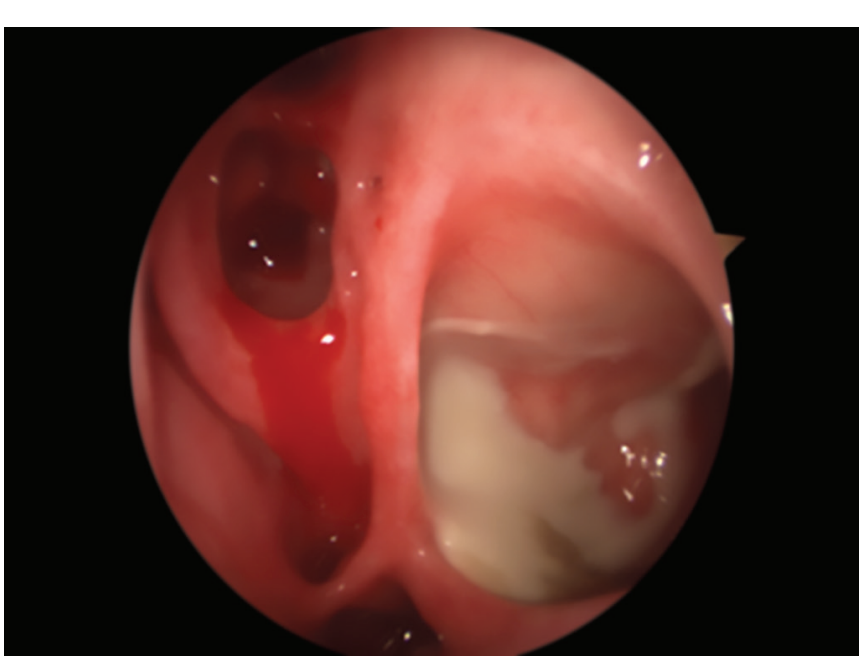

Fig. 6: Surgical mega-antrostomy with purulent sinus contents

"mega-antrostomies" in recalcitrant maxillary sinusitis. ${ }^{16,17}$ However, it should be noted that their series contained many patients with cystic fibrosis and previous CaldwellLuc procedures that may have already led to stagnation of mucociliary clearance and mean that the mega-antrostomy is a justifiable last resort when methods that are more conservative have failed. The mega-antrostomy is, in effect, a partial medial maxillectomy, as it involves removal of some of the inferior turbinate so that the ostium is lowered to the nasal floor and has been described as such elsewhere. ${ }^{18}$ Therefore, in select cases, as dictated by pathology or underlying systemic disease, there is certainly a case for making a larger antrostomy, ${ }^{19}$ but adherence to the Messerklinger principles above for the uncomplicated CRS patients should help to reduce the chances of failure in routine ESS.

\section{Medial Maxillectomy with or without a Transseptal Approach}

Typically, the medial maxillectomy is described for access to the maxillary sinus in order to remove lesions, such as an inverted papilloma, the most common sinonasal form of neoplasia. In the era of the endoscope, endoscopic medial maxillectomy has replaced traditional open techniques, such as the lateral rhinotomy and midfacial degloving approaches and has brought with it better results and reduced morbidity. ${ }^{20-22}$ Given that the natural ostium of the maxillary sinus lies close posteriorly to the nasolacrimal duct, removal of the medial sinus wall will often involve sacrificing the nasolacrimal duct and part of the inferior turbinate. In order to minimize the functional impact of the procedure, various modifications have been established. ${ }^{23,24}$ Other surgeons have advocated a more conservative pedicle-oriented surgical strategy. ${ }^{25}$ Key to planning the best approach is ensuring that both high-resolution computed tomography and magnetic resonance imaging are obtained. This will not only help direct the surgeon to the tumor attachment site but will better differentiate tumor from retained mucus. Tumors attached in the anterior part of the maxillary sinus pose a more difficult conundrum when performing an endoscopic medial maxillectomy as the site of attachment is $90^{\circ}$ to the plane of instrumentation and passage of the endoscope. Whilst it is possible to utilize angled endoscopes and microdebrider blades and drill bits, this area will remain difficult to access from the ipsilateral nasal cavity. Some will resort to a Caldwell-Luc in this scenario, but this will usually mean entering the sinus on the attachment site. A way around this is to utilize a transseptal approach from the contralateral nostril. By creating a staggered incision across the nasal septum, an angled debrider blade or drill bit can be passed through the septum and brought to bear on the site of attachment of the tumor.

\section{Prelacrimal Approaches to the Maxillary Sinus}

Another alternative approach to accessing difficult pathology in the maxillary sinus has been advocated more recently. The prelacrimal approach has been described by several authors (Fig. 7). ${ }^{26-29}$ Typically, it involves a vertical incision at the front of the inferior turbinate on the lateral wall of the nose. After raising a mucosal flap over the bone, the medial maxillary wall can then be entered anterior to the nasolacrimal duct using an osteotome or drill. Access to the sinus can be further widened by moving the inferior turbinate/maxillary medial wall complex medially into the nasal cavity. Additional access can be gained through the piriform aperture and anterior sinus wall openings. The advantage to this technique is direct access to anterior wall of the maxillary sinus without repositioning or excision of the nasolacrimal duct. Furthermore, the inferior turbinate/ maxillary medial complex can be returned to original anatomical status at the end of the process reducing the functional morbidity associated with the removal of the lateral nasal wall. These advantages are negated if the pathology directly involves the medial maxillary wall and necessitates its resection as part of the surgical plan.

A similar technique of canine fossa trephine can also allow access into the maxillary sinus without the need for radical maxillary medial wall resection for access. The technique uses the traditional oral canine puncture site without the need for incision or dissection but instead by a trephine portal that allows scope and instruments to be introduced without the morbidity of the traditional Caldwell-Luc approach. ${ }^{30}$

\section{DISCUSSION}

The approach chosen for endoscopic surgery of the maxillary sinus will depend on the pathology, goal of the surgery, skill and experience of the surgeon, and the equipment available to them. Acquiring the necsary skills 


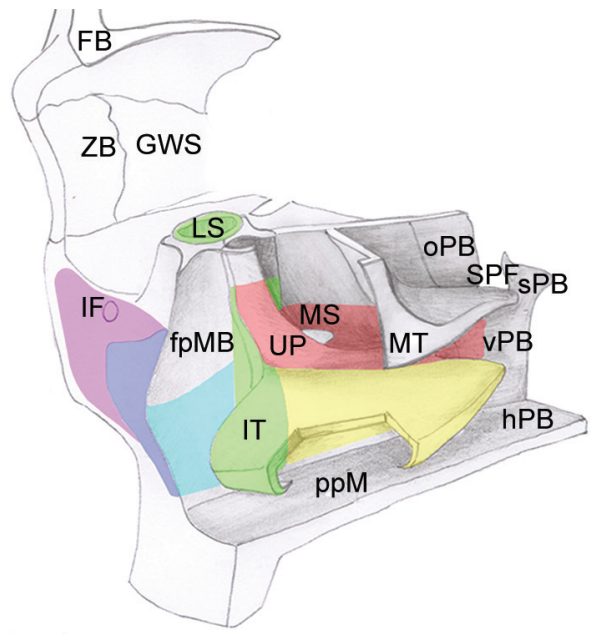

A

B
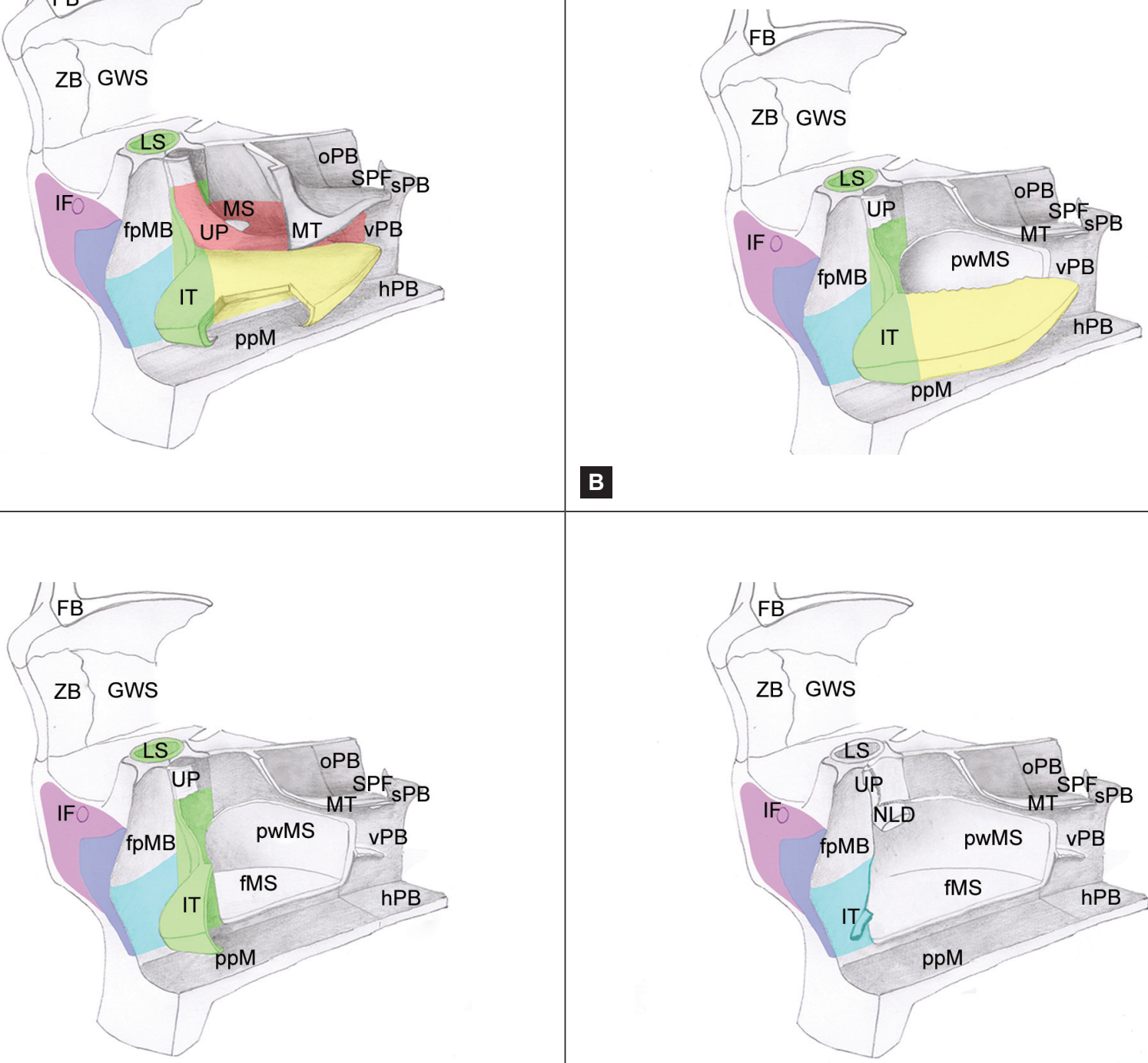

c

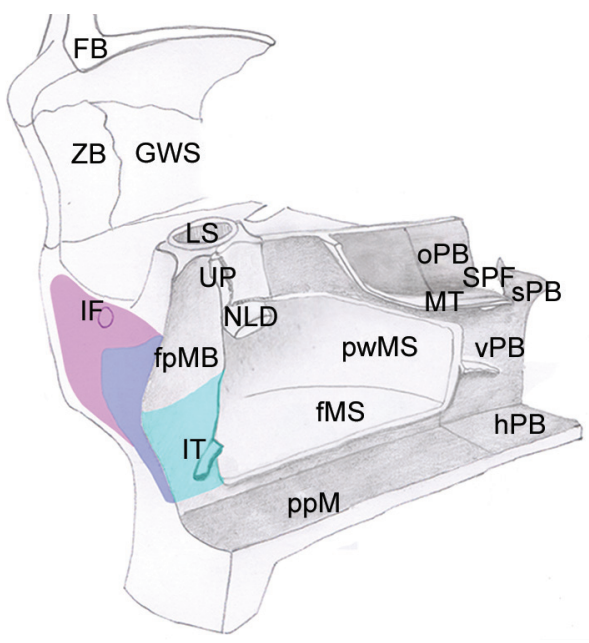

D

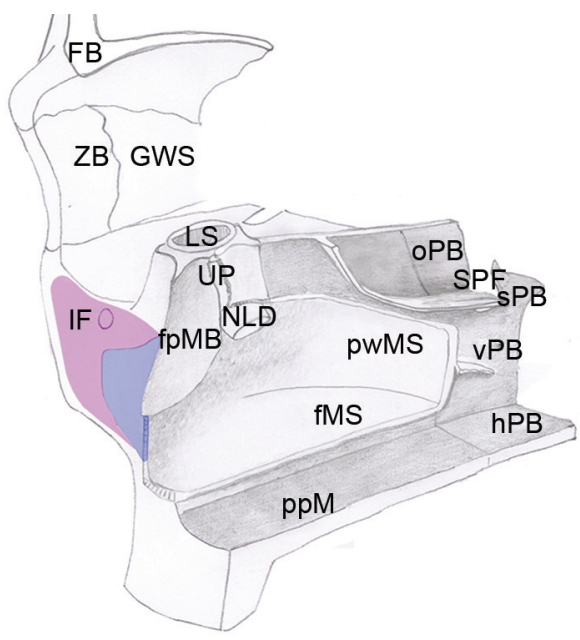

E

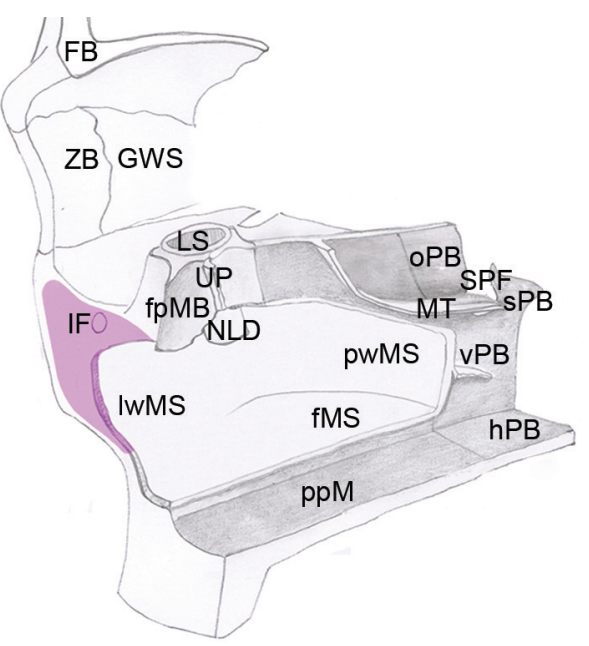

$\mathbf{F}$

Figs 7A to F: The transnasal endoscopic classification of partial maxillectomy as proposed by Turri-Zanoni et al. ${ }^{26}$ 
in all of these aspects may not routinely be accessible in all training programs and trainees should be encouraged to seek subspecialist fellowship opportunities at tertiary centers. Nontraining grades and established practitioners may also seek opportunities to develop new skills or enhance old ones through accredited cadaveric dissection courses.

\section{CONCLUSION}

From balloon sinuplasty to endoscopic medial maxillectomy, endonasal approaches to the maxillary sinus enable otorhinolaryngologists to manage varying pathologies within the maxillary sinus with minimal morbidity to the patient.

\section{REFERENCES}

1. Stammberger H, Posawetz W. Functional endoscopic sinus surgery. Concept, indications and results of the Messerklinger technique. Eur Arch Otorhinolaryngol $1990 \mathrm{Feb}$;47(2): 63-76.

2. Hopkins C, Browne JP, Slack R, Lund V, Topham J, Reeves B, Copley L, Brown P, van der Meulen J. The national comparative audit of surgery for nasal polyposis and chronic rhinosinusitis. Clin Otolaryngol 2006 Oct;31(5):390-398.

3. Bewick J, Egro FM, Masterson L, Javer AR, Philpott CM. Anatomic findings in revision endoscopic sinus surgery: case series and review of contributory factors. Allergy Rhinol (Providence) 2016 Jan;7(3):e151-e157.

4. Koskinen A, Penttila M, Myller J, Hammaren-Malmi S, Silvola J, Haahtela T, Hytonen M, Toppila-Salmi S. Endoscopic sinus surgery might reduce exacerbations and symptoms more than balloon sinuplasty. Am J Rhinol Allergy 2012 Nov-Dec;26(6):e150-e156.

5. Chaaban MR, Baillargeon JG, Baillargeon G, Resto V, Kuo YF. Use of balloon sinuplasty in patients with chronic rhinosinusitis in the United States. Int Forum Allergy Rhinol 2017 Jun;7(6):600-608.

6. Levine H, Rabago D. Balloon sinuplasty: a minimally invasive option for patients with chronic rhinosinusitis. Postgrad Med 2011 Mar;123(2):112-118.

7. Ahmed J, Pal S, Hopkins C, Jayaraj S. Functional endoscopic balloon dilation of sinus ostia for chronic rhinosinusitis. Cochrane Database Syst Rev 2011 Jul;(7):CD008515.

8. Bikhazi N, Light J, Truitt T, Schwartz M, Cutler J, Investigators RS. Standalone balloon dilation versus sinus surgery for chronic rhinosinusitis: a prospective, multicenter, randomized, controlled trial with 1-year follow-up. Am J Rhinol Allergy 2014 Jul-Aug;28(4):323-329.

9. Bizaki AJ, Numminen J, Taulu R, Rautiainen M. A controlled, randomized clinical study on the impact of treatment on antral mucociliary clearance: uncinectomy versus balloon sinuplasty. Ann Otol Rhinol Laryngol 2016 May;125(5):408-414.

10. Abreu CB, Balsalobre L, Pascoto GR, Pozzobon M, Fuchs SC, Stamm AC. Effectiveness of balloon sinuplasty in patients with chronic rhinosinusitis without polyposis. Braz J Otorhinolaryngol 2014 Sep-Dec;80(6):470-475.

11. Awad Z, Bhattacharyya M, Jayaraj SM. Anatomical margins of uncinectomy in endoscopic sinus surgery. Int J Surg 2013 Jan;11(2):188-190.

12. Gore MR, Ebert CS, Zanation AM, Senior BA. Beyond the "central sinus": radiographic findings in patients undergoing revision functional endoscopic sinus surgery. Int Forum Allergy Rhinol 2013 Feb;3(2):139-146.

13. Kennedy DW, Adappa ND. Endoscopic maxillary antrostomy: not just a simple procedure. Laryngoscope 2011 Oct;121(10): 2142-2145.

14. Simmen, D.; Jones, N. Manual of endoscopic sinus and skull base surgery. Stuttgart: Thieme; 2014.

15. Kirihene RK, Rees G, Wormald PJ. The influence of the size of the maxillary sinus ostium on the nasal and sinus nitric oxide levels. Am J Rhinol 2002 Sep-Oct;16(5):261-264.

16. Cho DY, Hwang PH. Results of endoscopic maxillary megaantrostomy in recalcitrant maxillary sinusitis. Am J Rhinol 2008 Nov-Dec;22(6):658-662.

17. Costa ML, Psaltis AJ, Nayak JV, Hwang PH. Long-term outcomes of endoscopic maxillary mega-antrostomy for refractory chronic maxillary sinusitis. Int Forum Allergy Rhinol 2015 Jan;5(1):60-65.

18. Konstantinidis I, Constantinidis J. Medial maxillectomy in recalcitrant sinusitis: when, why and how? Curr Opin Otolaryngol Head Neck Surg 2014 Feb;22(1):68-74.

19. Thompson CF, Conley DB. What is the optimal maxillary antrostomy size during sinus surgery? Curr Opin Otolaryngol Head Neck Surg 2015 Feb;23(1):34-38.

20. Pagella F, Giourgos G, Matti E, Canevari FR, Carena P. Endoscopic treatment of maxillary inverted papilloma. Rhinology 2011 Aug;49(3):369-374.

21. Wormald PJ, Ooi E, van Hasselt CA, Nair S. Endoscopic removal of sinonasal inverted papilloma including endoscopic medial maxillectomy. Laryngoscope 2003 May;113(5): 867-873.

22. Jurado-Ramos A, Jodas JG, Romero FR, Linares EA, Del Castillo FM, Gomariz EM, Baños EC. Endoscopic medial maxillectomy as a procedure of choice to treat inverted papillomas. Acta Otolaryngol 2009 Sep;129(9):1018-1025.

23. Nakayama T, Asaka D, Okushi T, Yoshikawa M, Moriyama H, Otori N. Endoscopic medial maxillectomy with preservation of inferior turbinate and nasolacrimal duct. Am J Rhinol Allergy 2012 Sep-Oct;26(5):405-408.

24. Suzuki M, Nakamura Y, Nakayama M, Inagaki A, MurakamiS, Takemura K, Yokota M. Modified transnasal endoscopic medial maxillectomy with medial shift of preserved inferior turbinate and nasolacrimal duct. Laryngoscope 2011 Nov;121(11):2399-2401.

25. Landsberg R, Cavel O, Segev Y, Khafif A, Fliss DM. Attachmentoriented endoscopic surgical strategy for sinonasal inverted papilloma. Am J Rhinol 2008 Nov-Dec;22(6):629-634.

26. Turri-Zanoni M, Battaglia P, Karligkiotis A, Lepera D, Zocchi J, Dallan I, Bignami M, Castelnuovo P. Transnasal endoscopic partial maxillectomy: operative nuances and proposal for a comprehensive classification system based on 1378 cases. Head Neck 2017 Apr;39(4):754-766.

27. Lin YT, Lin CF, Yeh TH. Application of the endoscopic prelacrimal recess approach to the maxillary sinus in unilateral maxillary diseases. Int Forum Allergy Rhinol 2018 Apr;8(4): 530-536.

28. Morrissey DK, Wormald PJ, Psaltis AJ. Prelacrimal approach to the maxillary sinus. Int Forum Allergy Rhinol 2016 Feb;6(2): 214-218.

29. Zhou B, Han DM, Cui SJ, Huang Q, Wang CS. Intranasal endoscopic prelacrimal recess approach to maxillary sinus. Chin Med J (Engl) 2013 Apr;126(7):1276-1280.

30. Seiberling K, Ooi E, MiinYip J, Wormald PJ. Canine fossa trephine for the severely diseased maxillary sinus. Am J Rhinol Allergy 2009 Nov-Dec;23(6):615-618. 